\title{
Clinical drug trials in general practice: a 10-year overview of protocols
}

\author{
Anja Maria Brænd*, Kaspar Buus Jensen, Atle Klovning and Jørund Straand
}

\begin{abstract}
Background: Drugs predominantly prescribed in general practice should ideally be tested in that setting; however, little is known about drug trials in general practice. Our aim was to describe drug trials in Norwegian general practice over the period of a decade.

Methods: The present work concerns a 10-year retrospective study of protocols submitted to the Norwegian national medicines agency (1998 to 2007) identifying all studies involving general practitioners (GPS) as clinical investigator(s). We analyzed the number of trials, drug company involvement, patients, participating doctors, payment, medications tested and main diagnostic criteria for inclusion. We also analyzed one trial in greater detail.

Results: Out of 2,054 clinical drug trials, 196 (9.5\%) were undertaken in general practice; 93\% were multinational, $96 \%$ were industry funded and $77 \%$ included patients both from general practice and specialist care. The trials were planned to be completed in the period 1998 to 2012. A total of 23,000 patients in Norway and 340,000 patients internationally were planned to be included in the 196 trials. A median of 5 GPs participated in each trial (range 1 to 402). Only $0.7 \%$ of $831 \mathrm{GP}$ investigators had general practice university affiliations. Median payment for participating investigators was $€ 1,900$ (range $€ 0$ to 13,500) per patient completing the trial. A total of 30 pharmaceutical companies were involved. The drugs most commonly studied were antidiabetics (21\%), obstructive airway disease medications (12\%), agents acting on the renin-angiotensin system (10\%), and lipid modifying agents (10\%). One trial, presented in more detail, had several characteristics of a seeding or marketing trial.
\end{abstract}

Conclusions: Only one in four drug trials involving general practice were solely general practice trials and almost all were industry initiated without input from academic general practice. There was a large variation in the number of patients, participating doctors, and economic compensation for trial investigators, with some investigators receiving substantial payments.

Keywords: Clinical trial, Drug industry, General practice, Patient selection, Randomized controlled trials

\section{Background}

About $90 \%$ of all drug prescriptions for outpatients are issued in general practice [1]. Patients in general practice are more likely to have less severe disease, and more undifferentiated symptoms than selected patients in secondary or tertiary health care, however, multimorbidity is common [2]. If a study population in a drug trial differs significantly from the population where the drug is most likely to be prescribed, the external validity of the trial may be impaired [3]. To avoid this uncertainty, many have argued that more clinical drug trials should

\footnotetext{
* Correspondence: ambraend@medisin.uio.no

Department of General Practice, Institute of Health and Society, Faculty of Medicine, University of Oslo, PO Box 1130, Blindern, Oslo N-0318, Norway
}

be conducted in primary care settings to ensure that the benefits are proportionate to the risks and costs of the treatment for patients in general practice $[4,5]$. Clinical drug trials may serve different purposes, from research of effectiveness, to marketing [6]. Trials with the main purpose of introducing new drugs to prescribers are often referred to as 'seeding trials'. Various criteria for seeding trials have been proposed (Table 1) [7-9], and it is characteristic that the trials are designed to make many clinicians familiar with a new drug. Being frequent prescribers, general practitioners (GPs) are probably of particular interest for designers of seeding trials; this is also because GPs are relatively independent in their decision-making processes.

C Biomed Central

(c) 2013 Brænd et al.; licensee BioMed Central Ltd. This is an Open Access article distributed under the terms of the Creative Commons Attribution License (http://creativecommons.org/licenses/by/2.0), which permits unrestricted use, distribution, and reproduction in any medium, provided the original work is properly cited. 
Table 1 Case study: the 'On-demand Nexium Evaluation' (ONE) trial ${ }^{\text {a }}$

\begin{tabular}{|c|c|}
\hline Key characteristics of seeding trials [7-9] & Does this apply for the ONE trial? \\
\hline Tests a new drug recently or about to be licensed & Yes: application year 2000, study completed by the end of 2001; esomeprazole licensed in 2001 \\
\hline Many well established competing products & Yes: omeprazole from 1989, lansoprazole and pantoprazole from 1995 \\
\hline Many patients included & $\begin{array}{l}\text { Yes: } 2,500 \text { patients (the trial with most Norwegian patients to be included). In addition, similar } \\
\text { studies have been conducted in other countries (see main text). }\end{array}$ \\
\hline $\begin{array}{l}\text { Frequent prescribers in the role of clinical } \\
\text { investigators }\end{array}$ & Yes: 402 general practitioners (GPs; the study involving most Norwegian GP investigators) \\
\hline $\begin{array}{l}\text { Often redundant as they are not designed for } \\
\text { answering a scientific problem }\end{array}$ & Probably yes \\
\hline Unreasonably high payments for the investigators & No: $€ 750$ which is below the 25 th percentile for all trials \\
\hline Results are often not published & No: there were three publications \\
\hline Conducted by drug company marketing departments & Unknown \\
\hline
\end{tabular}

In the UK between 1984 and 1989, general practice trials initiated by pharmaceutical companies were judged to have a low output of clinically relevant results [10]. The concerns addressed in that audit were mainly payments to GPs, information and safety issues, shortfall of investigators and patients, leading to inconclusive results, and low publication rates [10]. Among general practice studies published 1991 to 1996 in the BMJ, the British Journal of General Practice, and Family Practice, only $6 \%$ were randomized controlled trials, which was pointed out as a major challenge for the general practice research community [11]. Some Norwegian data regarding clinical drug trials have previously been reported, based on research applications submitted to the Research Ethical Committees (REC) [12-14] and the Norwegian Medicines Agency (NoMA) [15,16]. Between $68 \%$ and $85 \%$ of the trials were initiated by pharmaceutical industry [13-15], however, neither of these reports highlighted studies in general practice.

From the 1970s, a subcommittee of the Norwegian College of General Practitioners had a voluntary agreement with the pharmaceutical industry to assess protocols for studies to be performed in general practice $[17,18]$. If the committee judged the trial to be clinically relevant and of sound scientific quality, GPs were recommended to participate in the trial. However, by the turn of the millennium, the committee voiced critical comments regarding some trials judged to be marketing (due to features of seeding trials) rather than research [17]. Since then, this voluntary quality and relevance check has been largely bypassed by the industry [19], and information regarding industry initiated research in Norwegian general practice has thus become an almost hidden reality except for the investigators themselves. This prompted the Norwegian College of General Practitioners in 2008 to fund research to gain systematic knowledge of the drug research carried out by GPs. This included establishing an overview of all trials conducted, exploring whether GPs were subject for marketing trials, and assessing the relevance of the research questions addressed, as well as the studies' validity and publication output. The College requested this knowledge due to possible implications for their policy for future professional development within the discipline of general practice.

The aim of the present study was to establish a descriptive basis regarding clinical drug trials conducted in general practice by research areas and trial characteristics. As most clinical drug trials are multinational, this study also should concern research in general practice outside of Norway.

\section{Methods}

In Norway, the only complete national archive for drug trials is held by NoMA. Based on a hand search of the NoMA archive for the 10-year period 1998 to 2007, we describe all clinical trials planned to be carried out in general practice by their funding, clinical setting, drugs, diagnoses, patients, clinical investigators and economic compensations for the investigators. More detail will be given regarding one of the trials in year 2000, which the Norwegian College of GPs discouraged GPs to join [17], to give the reader a better sense of what we consider a seeding trial. Ethical approval for our study was not required.

\section{Dataset}

In Norway, all clinical pharmaceutical trials regardless of setting need approval from NoMA, the national, regulatory authority for new and existing medicines. Clinical trials are regulated by international and national laws, in which there were amendments between 1998 and 2007, and from 2004 the European Directive 2001/20/EC has been implemented in the Norwegian regulation [16]. By 
hand searching NoMA's total paper archive, protocol by protocol, we were able to identify application forms, protocols and other correspondence received by NoMA regarding applications for clinical drug trials between 1998 and 2007, the 10-year period before conversion to electronic registration. The computed total number of trial applications during this decade was 2,054 [16,20] (personal communication from I. Aaløkken, NoMA). The main hand search was performed in May to August 2008 by KBJ. Supplementary data collection and verification was performed by AMB between November to December 2011 for a total of 89 studies. No formal inter-rater reliability calculations were performed, but no significant errors or discrepancies were disclosed. A random check of non-included studies in the archive did not reveal any additional general practice trials. Applications for trials planned to take place solely or partly in general practice were included. General practice trials were defined as trials where the address and/or titles indicated that at least one of the Norwegian clinical investigators worked in general practice.

\section{Variables: trial characteristics}

For included studies, we recorded study title, medication tested in terms of its Anatomical Therapeutic Chemical (ATC) classification code [21], funders, whether the study was planned exclusively to be conducted in general practice or also in specialist care settings, whether it was a national or international study, and trial phase based on explicit information in the application ((I) study of toxicity and side effects; (II) study of doseresponse; (III) comparison with established treatment or placebo; or (IV) post-marketing study, gaining broader experience) [6]. We defined a study as industry initiated when a pharmaceutical company funded the study, wrote the study protocol or conducted the study, either the company itself or via a contract research organization. If the study drugs were provided free of charge, but the trial was otherwise designed and conducted independently under the responsibility of a GP researcher, it was defined as researcher initiated. There were no trials with unclear funding. We recorded the number of patients planned to be included internationally, and at Norwegian trial sites, the number of clinical investigators involved, trial duration, and if available, economic compensation for physicians involved. We also identified clinical investigators who were GP academics, that is, having affiliations to Norwegian general practice university departments. The main diagnostic criteria for inclusion in a study were based on the study title, and when necessary supplemented with information from the protocol and categorized according to the International Classification of Primary Care, second edition (ICPC-2) [22]. One of the authors (AMB) performed the categorization, and when in doubt, consensus was reached through discussions between $\mathrm{AK}$, JS and AMB.

\section{Data analysis}

Data were compiled in a spreadsheet. To compare planned number of patients to be enrolled in trials partly or entirely to be undertaken in general practice, we used the independent samples Mann-Whitney $U$ test, and otherwise we used descriptive statistics. Due to data skewness, median values are presented rather than means. We roughly estimated investigator incomes by multiplying median payment and median number of patients planned recruited divided by median number of investigators. Statistical analyses were performed using SPSS statistics (PASW Statistics 18; SPSS Inc., Chicago, IL, USA).

\section{Case study}

One outlying case is described in greater detail. For this trial, additional information is given on patients, interventions, comparison and outcome based on the study protocol. A systematic literature search was performed to identify publications arising from the trial (date of literature search, 17 September 2012). The trial was assessed according to the proposed criteria for seeding trials outlined in Table 1 [7-9].

\section{Results}

\section{Dataset: general practice drug trials}

During the 10-year period of applications, a total of 196 studies (9.5\% of all 2,054 clinical drug trials) were planned to be conducted entirely or partly in a general practice setting. Of these, 189 (96.4\%) trials were industry initiated and $7(3.6 \%)$ were researcher initiated (Table 2). The trials were planned to be completed in the period 1998 to 2012 . Figure 1 shows that the majority of the 196 trials had trial sites both in general practice and in specialist care. The majority of the trials were multinational. Only 45 trials ( $2.2 \%$ of all) were planned conducted entirely in general practice. The number of participating countries per trial varied from 1 to 50 (median: 9, interquartile range 5 to 17); however, for 112 trials information regarding the number of countries was incompletely stated.

\section{Trial characteristics}

A total of 30 different pharmaceutical companies applied for drug trials in general practice during this 10-year period. The initiators (number of trials) were GlaxoSmithKline (39), AstraZeneca (32), Novartis (21), MSD (19), Pfizer (11), Novo Nordisk (9), Boehringer Ingelheim (8), Roche (7), Lundbeck (5), and Schering-Plough (4). In addition, 5 companies initiated 3 trials, 4 companies initiated 2 trials, and 11 companies initiated 1 trial. The top 
Table 2 Clinical drug trial applications in Norway 1998 to 2007

\begin{tabular}{|c|c|c|c|c|c|c|}
\hline \multirow[t]{2}{*}{ Year } & \multicolumn{3}{|c|}{ General practice trials } & \multicolumn{3}{|c|}{ All clinical trials } \\
\hline & Industry initiated (GP only) & Researcher initiated $^{a}$ & Total (GP only) & Industry initiated & Researcher initiated & Total \\
\hline 1998 & $17(7)$ & 0 & $17(7)$ & 186 & 50 & $236^{\mathrm{b}}$ \\
\hline 1999 & $16(6)$ & 0 & $16(6)$ & 161 & 28 & $189^{c}$ \\
\hline 2000 & $23(10)$ & 1 & $24(11)$ & 173 & 65 & $238^{d}$ \\
\hline 2001 & $22(1)$ & 0 & $22(1)$ & 158 & 62 & $220^{d}$ \\
\hline 2002 & $16(5)$ & 2 & $18(7)$ & 143 & 50 & $193^{d}$ \\
\hline 2003 & $14(1)$ & 1 & $15(2)$ & 151 & 50 & $201^{d}$ \\
\hline 2004 & $22(2)$ & 1 & $23(3)$ & 171 & 60 & $231^{d}$ \\
\hline 2005 & $18(2)$ & 1 & $19(3)$ & 139 & 55 & $194^{d}$ \\
\hline 2006 & $20(1)$ & 1 & $21(2)$ & 113 & 60 & $173^{d}$ \\
\hline 2007 & 21 (3) & 0 & 21 (3) & 126 & 53 & $179^{d}$ \\
\hline Total & 189 (38) & 7 & $196(45)$ & 1,521 & 533 & 2,054 \\
\hline Mean/year & 18.9 (3.8) & 0.7 & $19.6(4.5)$ & 152.1 & 53.3 & 205.4 \\
\hline
\end{tabular}

Applications at the Norwegian Medicines Agency (NoMA) for clinical drug trials conducted entirely (general practitioners (GPs) only) or partly in general practice identified by hand searching of all the clinical trials in the archive. For comparison, an overview of all clinical trials is included.

${ }^{a}$ All researcher-initiated trials were GP only.

bersonal communication from Ingvild Aaløkken, Head of section, Preclinical assessment and clinical trials, NoMA, 2008.

'See [16].

${ }^{\mathrm{d}}$ See [20].

5 companies in terms of Norwegian market share [23] were responsible for 107 (55\%) of all trials.

Table 3 shows the number of patients and physicians involved and estimated economic compensation for the investigators. More than 23,000 Norwegian patients were to be included in the 196 trials, and almost 340,000 patients

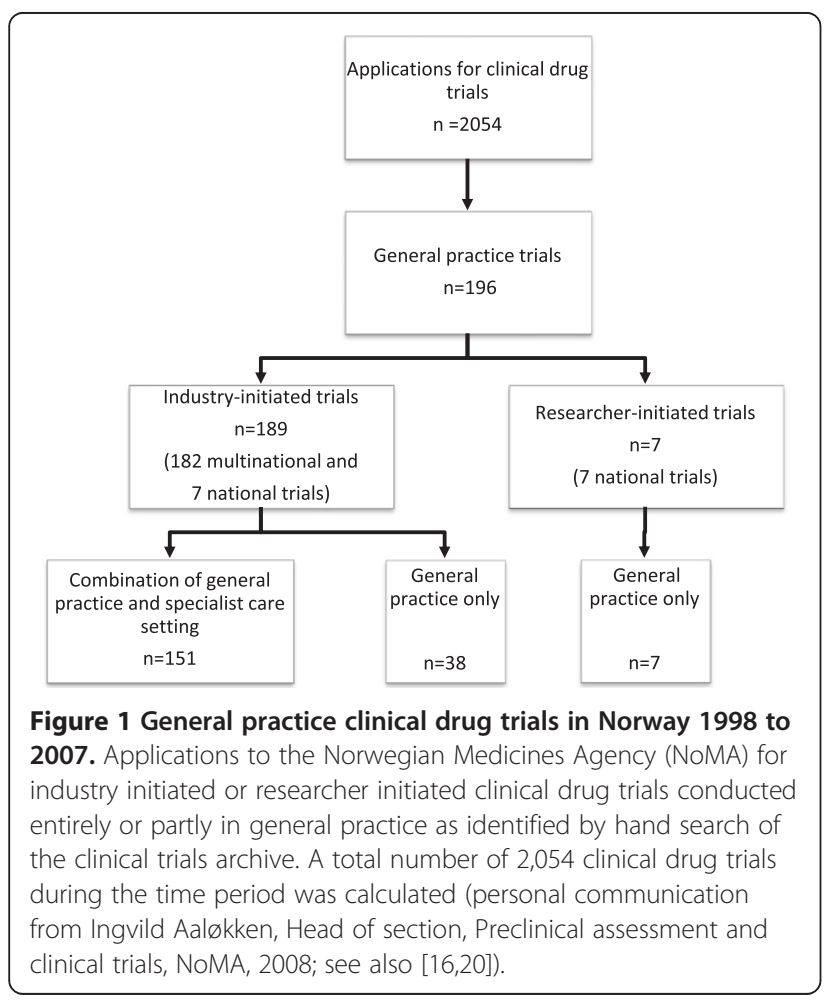

included internationally. In 6 trials the planned number of included patients was more than 10,000, and in 10 trials the number was less than 100 patients. The recruitment targets did not differ between trials in general practice only and those undertaken in mixed health care settings $(P=0.91)$. Only $6(0.7 \%)$ out of 831 clinical investigators were general practice academics, 3 of whom were only involved in trials without commercial sponsors. Information regarding trial investigators' payment was missing in 90 applications, 73 of which were from the period 1998 to 2002.

We were able to record the study phase in 122 trials, out of which none were phase I studies, $11 \%$ were phase II studies, $61 \%$ phase III and $27 \%$ phase IV studies.

Drugs from 30 different therapeutic groups were investigated in the trials (Table 4). The largest groups were antidiabetics, drugs for obstructive airway diseases, agents acting on the renin-angiotensin system, and lipid modifying agents. The top 5 therapeutic subgroups represented 121 (59\%) of all drug groups tested, the top 10 represented $158(78 \%)$. Only one of the trials investigated medication discontinuation.

The main diagnostic inclusion criteria represented 44 different diagnoses (Table 5), the top 5 of which made up 114 ( $52 \%$ of the inclusion criteria) and the top 10 made up 146 (67\%). In 14 trials no diagnosis was applicable, that is, healthy people, subjects over a certain age, smokers, and patients using baby aspirin.

\section{Case study}

In Table 1, more detail is given for one particular trial which the Norwegian College of GPs discouraged GPs to 
Table 3 Characteristics for clinical drug trials in general practice

\begin{tabular}{|c|c|c|c|c|c|c|c|}
\hline & \multicolumn{3}{|c|}{ Patients planned for inclusion } & \multicolumn{2}{|c|}{ Clinical investigators } & \multicolumn{2}{|c|}{ Payment } \\
\hline & Norway & $\begin{array}{c}\text { All } \\
\text { countries }\end{array}$ & $\begin{array}{c}\text { Trial duration, } \\
\text { weeks }\end{array}$ & $\begin{array}{l}\text { General } \\
\text { practice }^{a}\end{array}$ & $\begin{array}{l}\text { All sites in } \\
\text { Norway }\end{array}$ & $\begin{array}{l}\text { Investigators' payment per } \\
\text { patient, } \epsilon^{\mathrm{b}}\end{array}$ & $\begin{array}{c}\text { Estimated yearly income per } \\
\text { investigator, } €^{\mathrm{c}}\end{array}$ \\
\hline Median & 60 & 672.5 & 24 & 5 & 7 & 1,900 & 1,600 \\
\hline $\begin{array}{l}\text { Minimum to } \\
\text { maximum }\end{array}$ & 8 to 2,500 & 8 to 31,000 & 1 to 288 & 1 to 402 & 1 to 402 & 0 to 13,500 & 0 to 59,000 \\
\hline $\begin{array}{l}2.5 \text { to } 97.5 \\
\text { percentile }\end{array}$ & 13 to 560 & 50 to 14,368 & 3 to 240 & 1 to 37 & 1 to 61 & 0 to 7,500 & 0 to 25,000 \\
\hline Sum & 23,635 & 337,921 & & 1,920 & 2,475 & & \\
\hline
\end{tabular}

This table shows trial characteristics of applications at the Norwegian Medicines Agency (NoMA), 1998 to 2007, for 196 clinical drug trials conducted solely or partly in general practice identified by hand searching of the archive.

${ }^{a}$ General practitioners (GPs) participated in clinical trials from 1 to 36 times, with median 1 and interquartile range 1 to 2 . A total of 831 unique GPs participated. b Information missing in 90 applications.

${ }^{c}$ Calculated using median payment and median number of patients recruited per investigator. This payment covers all expenses connected with the trial for the investigator.

join [17], the 'On-demand Nexium Evaluation' trial, with the following clinical characteristics. Patients: patients with symptoms suggestive of gastroesophageal reflux disease (GERD; heartburn with or without acid regurgitation) for 3 days or more were included. Only patients with effect of treatment with esomeprazole 40 mg were randomized for comparison with ranitidine. Intervention: the drug tested was esomeprazole 40/20 mg daily. Comparison: there was initially no comparison; if treatment success in the run-in period with esomeprazole $40 \mathrm{mg}$ daily, comparison was esomeprazole $20 \mathrm{mg}$ daily on demand or ranitidine $150 \mathrm{mg}$ twice daily. Outcomes: difference in direct medical costs (mean per patient) was the primary outcome, secondary objectives included health care contacts, tests and procedures, hospitalizations, patient time and travel costs, early retirement, absence from work, symptom registration, quality of life, self-perceived overall treatment effect, and patient satisfaction. Among all 196 trials in Norwegian general practice during the decade, this trial was designed to include the largest number of national GP investigators and patients. In the sample size calculations a power of $95 \%$ (beta $=0.05$ ) was used. The significance level alpha was $5 \%$. The study had an open design, with no blinding. There was a run-in period before randomization, and only patients responding to the high dose esomeprazole treatment were randomized and included in the intention-to-treat analyses. Per protocol analyses were also used. Three journal articles presenting results from the study were identified [24-26].

\section{Discussion}

The main findings in this study were that only about onetenth of all clinical drug trials in Norway involved GPs as clinical investigators recruiting patients from their practices, and only $3.6 \%$ of these were non-industry trials.

We found that the proportion of researcher-initiated clinical drug trials in general practice in Norway was minute (3.6\%) compared to clinical drug trials from other fields of medicine where $15 \%$ to $32 \%$ are noncommercial studies [13-15]. Conducting clinical trials in general practice poses some typical challenges, like difficulties with study logistics and patient recruitment in a large number of small trial sites [4]. The lack of researcher-initiated trials has also been pointed out in the European Research Agenda for General Practice [5]. Irrespective of their commercial interests, this research agenda underlines that drugs mainly targeting primary care should be appropriately tested in primary care settings. Furthermore, that a stable funding and formation of research networks may facilitate non-commercial randomized controlled trials in general practice [5]. Although many have pointed to the need for clinical drug trials conducted independently of the industry, there was nonetheless a decline in the number of non-commercial randomized controlled trials in the UK between 1980 and 2002 [27]. Several systematic reviews have pointed out that drug trials financed by pharmaceutical industry tend to publish results more in favor of the manufacturer's product than non-commercial trials, and that negative studies in this respect commonly remain unpublished [28-32]. This publication bias combined with the lack of non-industry trials in general practice might therefore contribute to an evidence-biased knowledge base.

Over the last few years, there has been a decline in the number of clinical trials in several European countries [33,34]. In Norway, the largest reduction has been in phase III studies [16]. For general practice studies we were not able to confirm this trend, even if the majority of trials in our material were phase III or IV. The trials where explicit information regarding trial phase was missing, were most likely also phase III or IV. The five companies funding most general practice trials were among the seven most profitable companies, thus largely reflecting their market positions [23]. 
Table 4 Anatomical Therapeutic Chemical classification (ATC code ${ }^{a}$ ) for test drugs in clinical drug trials in general practice

\begin{tabular}{|c|c|c|c|c|c|c|}
\hline Anatomical main ATC group & n & $\%$ & Therapeutic ATC subgroup & $\mathbf{n}$ & $\%$ & Rank (1 to 10 ) \\
\hline \multirow[t]{4}{*}{ A: Alimentary tract and metabolism } & \multirow[t]{4}{*}{52} & \multirow[t]{4}{*}{25.5} & A02: Drugs for acid-related disorders ${ }^{b}$ & 7 & 3.4 & \multirow[t]{4}{*}{7} \\
\hline & & & A03: Drugs for functional gastrointestinal disorders & 2 & 1.0 & \\
\hline & & & A08: Antiobesity preparations, excluding diet products & 1 & 0.5 & \\
\hline & & & A10: Drugs used in diabetes ${ }^{\mathrm{b}}$ & 42 & 20.6 & \\
\hline B: Blood and blood-forming organs & 4 & 2.0 & B01: Antithrombotic agents & 4 & 2.0 & \\
\hline \multirow[t]{4}{*}{ C: Cardiovascular system } & \multirow[t]{4}{*}{46} & \multirow[t]{4}{*}{22.5} & C02: Antihypertensives & 2 & 1.0 & \\
\hline & & & C08: Calcium channel blockers ${ }^{b}$ & 2 & 1.0 & \\
\hline & & & C09: Agents acting on the renin-angiotensin system & 21 & 10.3 & 3 \\
\hline & & & C10: Lipid modifying agents ${ }^{b}$ & 21 & 10.3 & 3 \\
\hline \multirow[t]{2}{*}{ D: Dermatologicals } & \multirow[t]{2}{*}{2} & \multirow[t]{2}{*}{1.0} & D01: Antifungals for dermatological use & 1 & 0.5 & \\
\hline & & & D04: Antipruritics, including antihistamines, anesthetics, etc. & 1 & 0.5 & \\
\hline \multirow[t]{3}{*}{ G: Genitourinary system and sex hormones } & \multirow[t]{3}{*}{8} & \multirow[t]{3}{*}{3.9} & G02: Other gynecologicals & 1 & 0.5 & \\
\hline & & & G03: Sex hormones and modulators of the genital system & 2 & 1.0 & \\
\hline & & & G04: Urologicals & 5 & 2.5 & 10 \\
\hline \multirow[t]{2}{*}{ H: Systemic hormonal preparations } & \multirow[t]{2}{*}{3} & \multirow[t]{2}{*}{1.5} & H02: Corticosteroids for systemic use ${ }^{c}$ & 2 & 1.0 & \\
\hline & & & H05: Calcium homeostasis & 1 & 0.5 & \\
\hline \multirow[t]{3}{*}{ J: Anti-infectives for systemic use } & \multirow[t]{3}{*}{22} & \multirow[t]{3}{*}{10.8} & J01: Antibacterials for systemic use ${ }^{b}$ & 4 & 2.0 & \\
\hline & & & J05: Antivirals for systemic use & 12 & 5.9 & 6 \\
\hline & & & J07: Vaccines & 6 & 2.9 & 9 \\
\hline L: Antineoplastic and immunomodulating agents & 2 & 1.0 & L01: Antineoplastic agents & 2 & 1.0 & \\
\hline \multirow[t]{2}{*}{ M: Musculoskeletal system } & \multirow[t]{2}{*}{15} & \multirow[t]{2}{*}{7.4} & M01: Anti-inflammatory and antirheumatic products & 13 & 6.4 & \multirow[t]{2}{*}{5} \\
\hline & & & M05: Drugs for treatment of bone diseases & 2 & 1.0 & \\
\hline \multirow[t]{5}{*}{ N: Nervous system } & \multirow[t]{5}{*}{23} & \multirow[t]{5}{*}{11.3} & N02: Analgesics & 7 & 3.4 & \multirow[t]{5}{*}{7} \\
\hline & & & N04: Anti-Parkinson drugs & 2 & 1.0 & \\
\hline & & & N05: Psycholeptics ${ }^{c}$ & 5 & 2.5 & \\
\hline & & & N06: Psychoanaleptics ${ }^{c}$ & 5 & 2.5 & \\
\hline & & & N07: Other nervous system drugs & 4 & 2.0 & \\
\hline \multirow[t]{3}{*}{ R: Respiratory system } & \multirow[t]{3}{*}{26} & 12.7 & R01: Nasal preparations & 1 & 0.5 & \\
\hline & & & R03: Drugs for obstructive airway diseases ${ }^{c}$ & 24 & 11.8 & 2 \\
\hline & & & R06: Antihistamines for systemic use & 1 & 0.5 & \\
\hline Missing information & & & & 1 & 0.5 & \\
\hline Total number of ATC codes & & & & 204 & 100 & \\
\hline
\end{tabular}

This table shows the ATC codes of drug being tested identified from applications to the Norwegian Medicines Agency (NoMA) for 196 clinical drug trials between 1998 and 2007 conducted entirely or partly in general practice through hand searching the archive.

${ }^{\mathrm{a} S e e}[21]$.

${ }^{\mathrm{b}}$ Additional ATC code in a total of eight trials with two ATC codes registered.

'ATC code in researcher-initiated trials; for the groups $\mathrm{H} 02$, N05 and R03 there were two trials.

A study from 2000 found that $84 \%$ of GPs in a part of England had been involved in research, $48 \%$ mainly in clinical trials [35]. Our figures indicate that Norwegian GPs' participation rate in clinical trials is less than half of that in the UK; the 831 GPs involved correspond to about $20 \%$ of all Norwegian GPs in 2002 [1]. Surprisingly few GPs with university affiliations had participated in clinical drug trials, although many of them work part-time as GPs and are particularly interested in clinical research. Explanations for this finding may be that they judged the clinical relevance of the industry funded trials to be low, feared conflicts of interest, or had general negative attitudes towards collaboration with pharmaceutical companies [36]. Most GPs involved in clinical trials only participated once or just a few times, and did therefore not gain large incomes for participating. However, a number of GPs were involved in quite a few trials, giving them a substantial yearly income from trial participation. A few trials also gave outstandingly high 
Table 5 Main diagnostic criteria for inclusion classified in terms of International Classification of Primary Care (ICPC) diagnoses $^{\mathrm{a}}$ for clinical drug trials

\begin{tabular}{|c|c|c|c|}
\hline ICPC diagnosis & $\mathrm{n}$ & $\%$ & $\begin{array}{l}\text { GP } \\
\text { only }\end{array}$ \\
\hline T90: Diabetes, non-insulin dependent & 44 & 20.2 & 1 \\
\hline K86: Hypertension, uncomplicated & 22 & 10.1 & 7 \\
\hline R96: Asthma & 19 & 8.7 & 4 \\
\hline T93: Lipid disorder & 18 & 8.3 & 2 \\
\hline R80: Influenza & 11 & 5.0 & 7 \\
\hline L89: Osteoarthrosis of hip & 7 & 3.2 & 2 \\
\hline L90: Osteoarthrosis of knee & 7 & 3.2 & 3 \\
\hline N89: Migraine & 7 & 3.2 & 1 \\
\hline R95: Chronic obstructive pulmonary disease & 6 & 2.8 & 2 \\
\hline P76: Depressive disorder & 5 & 2.3 & 2 \\
\hline K78: Atrial fibrillation/flutter & 4 & 1.8 & \\
\hline L88: Rheumatoid/seropositive arthritis & 4 & 1.8 & \\
\hline T82: Obesity & 4 & 1.8 & \\
\hline D07: Dyspepsia/indigestion & 3 & 1.4 & 2 \\
\hline P70: Dementia & 3 & 1.4 & 2 \\
\hline T99: Endocrine/metabolic/nutritional disease, other & 3 & 1.4 & \\
\hline D84: Oesophagus disease & 2 & 0.9 & 2 \\
\hline D93: Irritable bowel syndrome & 2 & 0.9 & 1 \\
\hline L84: Back syndrome without radiating pain & 2 & 0.9 & 1 \\
\hline L91: Osteoarthrosis, other & 2 & 0.9 & 1 \\
\hline L95: Osteoporosis & 2 & 0.9 & \\
\hline N04: Restless legs & 2 & 0.9 & \\
\hline P06: Sleep disturbance & 2 & 0.9 & \\
\hline R78: Acute bronchitis/bronchiolitis & 2 & 0.9 & 2 \\
\hline U04: Incontinence, urine & 2 & 0.9 & \\
\hline Y07: Impotence, not otherwise specified & 2 & 0.9 & \\
\hline A23: Risk factor, not otherwise specified & 1 & 0.5 & 1 \\
\hline $\begin{array}{l}\text { A91: Abnormal result investigation, not otherwise } \\
\text { specified }\end{array}$ & 1 & 0.5 & \\
\hline D12: Constipation & 1 & 0.5 & \\
\hline K76: Ischemic heart disease without angina & 1 & 0.5 & \\
\hline K99: Cardiovascular disease, other & 1 & 0.5 & \\
\hline L29: Symptom/complaint, musculoskeletal, other & 1 & 0.5 & \\
\hline L92: Shoulder syndrome & 1 & 0.5 & 1 \\
\hline L93: Tennis elbow & 1 & 0.5 & 1 \\
\hline L99: Musculoskeletal disease, other & 1 & 0.5 & \\
\hline R28: Limited function/disability (respiratory) & 1 & 0.5 & \\
\hline R75: Sinusitis, acute/chronic & 1 & 0.5 & 1 \\
\hline R76: Tonsillitis, acute & 1 & 0.5 & 1 \\
\hline R81: Pneumonia & 1 & 0.5 & \\
\hline R97: Allergic rhinitis & 1 & 0.5 & \\
\hline
\end{tabular}

Table 5 Main diagnostic criteria for inclusion classified in terms of International Classification of Primary Care (ICPC) diagnoses $^{\mathbf{a}}$ for clinical drug trials (Continued)

\begin{tabular}{llll}
\hline S74: Dermatophytosis & 1 & 0.5 & 1 \\
T89: Diabetes, insulin dependent & 1 & 0.5 & \\
X11: Menopausal symptom/complaint & 1 & 0.5 & \\
No diagnosis applicable & 14 & 6.4 & 2 \\
Total $^{\text {b }}$ & 218 & 100
\end{tabular}

Table shows main inclusion criteria classified as ICPC diagnoses identified from applications at the Norwegian Medicines Agency (NoMA) for 196 clinical drug trials between 1998 to 2007 conducted entirely (general practitioner (GP) only) or partly in general practice through hand searching of the archive. ${ }^{\text {a }}$ See [22].

${ }^{\mathrm{b}} \mathrm{A}$ total of 22 studies had 2 or more diagnoses as inclusion criteria; 3 studies had 3 diagnoses.

payment for the doctors involved. Most GPs in Norway are self-employed with a major part of their income from patient payment and per capita reimbursement. It is therefore reasonable that extra workload due to participation in clinical trials is compensated. But how much is a reasonable level of compensation? Payment to clinicians for participation in clinical trials varies a lot [36,37]. Andersen et al. report payments of US $\$ 800$ per patient enrolled for a Danish industry initiated trial [37], which is approximately one-third of the median payment in our material. Raftery et $a l$. point to the striking lack of transparency in guidelines for payments for involvement in research, where GPs are among the few individual clinicians where direct personal payment still is common [36]. Concerns have been voiced that high payments may create conflicts of interest and possibly lead to unethical recruitment processes [36]. This demands rigorous ethical standards from both the general practice community and the pharmaceutical industry.

We identified antidiabetic drugs and type II diabetes to be studied most frequently. Thomas et al. found few published articles on diabetes in UK primary care journals in the 1990s [11]. The general use of antidiabetics in Norway increased by $27 \%$ (in defined daily doses (DDDs)) from 2004 to 2008 [38]. This may both reflect increasing diabetes prevalence but also the introduction of new drugs. Andersen et al. found that physicians conducting a clinical trial, significantly increased the sponsor's share of prescribed drugs for the disease compared to GPs not involved in the trials [37], and this might be important for expanding the market. The many post-marketing studies of new and expensive insulin analogues with large market potentials have recently been criticized for limited scientific value [9]. The three largest drug groups investigated in our study were among the four most consumed drugs in Norway (in terms of DDDs) [38]. These therapeutic areas were also among the six most frequently researched topics in general practice internationally 
between 2003 and 2008 [5]. In Norway in years 2000 and 2004 [14], and in the UK 1980 to 2002 [27], most clinical trials were in the field of cancer and antineoplastic and immunomodulating drugs. We only identified two trials in this field. This was as expected, as GPs rarely initiate antineoplastic drug prescriptions. Only one trial studied the effects of discontinuation of (unnecessary) medication use, although this is an important topic both for quality of care and general practice research [5].

\section{Strengths and limitations of the study}

The NoMA archive is a mandatory, complete national archive of all clinical drug trials in Norway, and it is a strength that we managed to include all trial protocols, not restricted to a specific region or to trials that had been reported in scientific publications. The large proportion of multinational trials identified also increases the external validity of our findings, which therefore may be relevant for general practice in other countries. As the identification of trials was performed by hand searching, random errors may have occurred during this process and recording of results. The main hand search was performed by only one of the authors, but supplementary searches in the archive for completing information by another author did not reveal significant errors. The total number of trials (2,054 trials) for the 10-year period was summed up from numbers provided by NoMA, and the exact total number of investigated paper files was not recorded, although the whole archive was searched shelf by shelf. Some uncertainty regarding missing files in the archive is therefore possible, however, we believe the number of missing files to be negligible due to strict control and restricted access to the state run archive. We have not addressed the number of clinical trials that were never conducted, either from lack of approval from REC or NoMA, or for other reasons, but this will be a subject of future research. The patient numbers reported involve all trial sites in Norway, and we do not know the exact number of general practice patients. Concerning trial phase, the proportion of missing data was quite large. The same applies to investigator payments, which were underreported to NoMA, especially during the first years in the time period studied.

\section{Case study: a seeding trial?}

The intentions for conducting a seeding trial are in general hidden and they may therefore be difficult to identify. Seeding trials have usually been disclosed based on documents from litigation processes $[39,40]$. Without access to internal communication within the drug company, the judgment of whether a trial is designed for marketing has to be based on several aspects. The esomeprazole trial described has many features being typical for a seeding trial, with several of the key characteristics described in Table 1 [7-9].

Esomeprazole was launched in 2001 in a crowded drug group. It is the most expensive of the proton pump inhibitors, and had become the tenth most-sold medicine in Norway in 2008 calculated in terms of pharmacy retail prices [38]. The esomeprazole trial described was large, and the sample size calculations were based on a higher power than conventionally used [41]. A higher power demands larger sample sizes and more patients to be enrolled into the study. No ethical considerations regarding these issues were discussed in the peer-reviewed publications arising from the trial [24-26]. The open design and the inclusion only of patients responding to high doses of esomeprazole in the intention-to-treat analyses increased the risk of bias in favor of esomeprazole. One may question the scientific need for recruiting around 400 GPs in the trial, each GP only enrolling a handful of patients with gastroesophageal reflux, a relatively common health complaint. The large number of participating doctors was not explained or justified in the protocol or in publications arising from the trial [24-26]. Nevertheless, the trial was approved by both the regional ethics committee and NoMA without any major remarks. The general practice research committee criticized this protocol on several issues, some of which are listed above $[17,19,42]$. The trial was presented in Norway as a separate trial, but quite similar studies with the same protocol acronym (ONE) have been reported from Denmark (without ranitidine comparison) [43] and Switzerland [44] in total involving almost 3,500 patients.

\section{Conclusions}

Only one-tenth of all clinical drug trials in Norway involved patients recruited from general practice, and just one in four of these trials were solely general practice trials. Almost all trials were industry initiated without input from academic general practice. This shows that it is a challenge for general practice to increase the number of clinical trials in general and researcher initiated clinical drug trials in particular. Antidiabetic drugs were most commonly studied. There was a large variation in the number of patients, participating doctors, and economic compensation for trial investigators, with some investigators receiving substantial payments. We describe a study with several characteristics of a seeding trial, where none of the official approving bodies for clinical trials had any major remarks. It raises the important ethical considerations with regard to exposing patients to unnecessary risks in either an inordinately large or a small, underpowered trial.

In this descriptive study, we have addressed neither the relevance of the research questions for general practice nor the methodological quality or publication output of 
the trials beyond the case study. These are important issues that we intend to explore in forthcoming research.

\section{Abbreviations \\ ATC code: Anatomical Therapeutic Chemical classification code; DDD: Defined daily dose; ICPC: International Classification of Primary Care; GP: General practitioner; NoMA: Norwegian Medical Agency.}

\section{Competing interests}

The authors declare that they have no competing interests. The authors alone are responsible for the content and writing of the paper.

\section{Authors' contributions}

AMB completed the data collection, analyzed and interpreted the data and drafted the manuscript. KBJ participated in the design of the study, acquired most of the data and critically revised the manuscript. AK analyzed and interpreted the data, and critically revised the manuscript. JS conceived and designed the study, participated in the analysis and interpretation, and critically revised the manuscript. All authors read and approved the final manuscript.

\section{Acknowledgements}

We thank Ingvild Aaløkken, Head of Section, for preclinical assessment and clinical trials at the Norwegian Medicines Agency for positive support, guidance and admission to the NoMA archive. We also thank Rune B Jakobsen for critically reviewing the manuscript. This study was funded by a research scholarship from The General Practice Research Fund from The Norwegian Medical Association.

Received: 5 October 2012 Accepted: 13 May 2013

Published: 1 June 2013

\section{References}

1. Hunskår S: Allmennmedisin [General Practice]. 2nd edition. Oslo, Norway: Gyldendal Norsk Forlag; 2003.

2. Barnett K, Mercer SW, Norbury M, Watt G, Wyke S, Guthrie B: Epidemiology of multimorbidity and implications for health care, research, and medical education: a cross-sectional study. Lancet 2012, 380:37-43.

3. Rothwell PM: External validity of randomised controlled trials: "to whom do the results of this trial apply?". Lancet 2005, 365:82-93.

4. Gagyor I, Bleidorn J, Wegscheider K, Hummers-Pradier E, Kochen MM: Practices, patients and (im)perfect data - feasibility of a randomised controlled clinical drug trial in German general practices. Trials 2011, 12:91.

5. Hummers-Pradier E, Beyer M, Chevallier P, Eilat-Tsanani $\mathrm{S}$, Lionis C, Peremans L, Petek D, Rurik I, Soler JK, Stoffers HE, Topsever P, Ungan M, van Royen P: Series: The research agenda for general practice/family medicine and primary health care in Europe. Part 4. Results: specific problem solving skills. Eur J Gen Pract 2010, 16:174-181.

6. Laake P, Benestad HB, Olsen BR: Research Methodology in the Medical and Biological Sciences. London, UK: Academic Press; 2007

7. Kessler DA, Rose JL, Temple RJ, Schapiro R, Griffin JP: Therapeutic-class wars - drug promotion in a competitive marketplace. N Engl J Med 1994, 331:1350-1353.

8. Ross JS, Gross CP, Krumholz HM: Promoting transparency in pharmaceutical industry-sponsored research. Am J Public Health 2012, 102:72-80.

9. Gale EA: Post-marketing studies of new insulins: sales or science? BMJ 2012, 344:e3974.

10. Wise $P$, Drury M: Pharmaceutical trials in general practice: the first 100 protocols. An audit by the clinical research ethics committee of the Royal College of General Practitioners. BMJ 1996, 313:1245-1248.

11. Thomas T, Fahey T, Somerset M: The content and methodology of research papers published in three United Kingdom primary care journals. Br J Gen Pract 1998, 48:1229-1232.

12. Winther FO, Hole OP: Scientific quality of clinical research. An analysis of 40 research projects in pharmacology/pharmacotherapy. Eur J Clin Pharmacol 1997, 51:351-354.

13. Hole OP, Winther FO, Straume B: Clinical research: the influence of the pharmaceutical industry. Eur J Clin Pharmacol 2001, 56:851-853.
14. Winther FO, Hole OP, Nitter-Hauge S: An analysis of the clinical development of drugs in Norway for the years 2000 and 2004: the influence of the pharmaceutical industry. Eur J Clin Pharmacol 2007, 63:909-912.

15. Sandaker L, Fjeld B, Reikvam A, Lislevand H, Madsen S: [Clinical trials in Norway - completion and reporting are not satisfactory]. Tidsskr Nor Laegeforen 2004, 124:2888-2890.

16. Bjørnhaug E, Aaløkken I: [Clinical trials in Norway the last 10 years]. Nor Farm Tidsskr 2010, 12:12-14.

17. Holtedahl KA, Meland E, Klovning A: [Critical questions to drug industry]. Tidsskr Nor Laegeforen 2001, 121:1403-1404.

18. Meland E: Research ethics - revisited. Scand J Prim Health Care 2003, 21:129-131.

19. Holtedahl $K A$, Meland E: Drug trials in general practice: time for a quality check before recruiting patients. BMJ 2007, 335:7.

20. Norwegian Medicines Agency: [Yearly report 2008]. [http://slv.no/ Om_Legemiddelverket/arsrapportar/Documents/ aarsrapport_legemiddelverket_2008_web_090604.pdf].

21. WHO Collaborating Centre for Drug Statistics Methodology: ATC/DDD Index. [http://www.whocc.no/atc_ddd_index/].

22. ICPC: International Classification of Primary Care 2nd edition, electronic version [http://www.kith.no/upload/2705/ICPC-2-English.pdf].

23. The Pharmaceutical Industry in Norway: Facts and Figures 2008. [http://www. Imi.no/english].

24. Hansen AN, Bergheim R, Fagertun H, Lund H, Moum B: A randomised prospective study comparing the effectiveness of esomeprazole treatment strategies in clinical practice for 6 months in the management of patients with symptoms og gastroesophageal reflux disease. Int J Clin Pract 2005, 59:665-671.

25. Hansen AN, Bergheim R, Fagertun H, Lund H, Wiklund I, Moum B: Longterm management of patients with symptoms of gastro-oesophageal reflux disease - a Norwegian randomised prospective study comparing the effects of esomeprazole and ranitidine treatment strategies on health-related quality of life in a general practitioners setting. Int J Clin Pract 2006, 60:15-22.

26. Hansen AN, Wahlqvist $P$, Jorgensen E, Bergheim R, Fagertun $H$, Lund $H$, Moum B: Six-month management of patients following treatment for gastroesophageal reflux disease symptoms - a Norwegian randomized, prospective study comparing the costs and effectiveness of esomeprazole and ranitidine treatment strategies in a general medical practitioners setting. Int J Clin Pract 2005, 59:655-664.

27. Chalmers I, Rounding C, Lock K: Descriptive survey of non-commercial randomised controlled trials in the United Kingdom, 1980-2002. BMJ 2003, 327:1017.

28. Lundh A, Sismondo S, Lexchin J, Busuioc OA, Bero L: Industry sponsorship and research outcome. Cochrane Database Syst Rev 2012, 12:MR000033.

29. Schott G, Pachl H, Limbach U, Gundert-Remy U, Lieb K, Ludwig WD: The financing of drug trials by pharmaceutical companies and its consequences: part 2: a qualitative, systematic review of the literature on possible influences on authorship, access to trial data, and trial registration and publication. Dtsch Arztebl Int 2010, 107:295-301.

30. Bekelman JE, Li Y, Gross CP: Scope and impact of financial conflicts of interest in biomedical research: a systematic review. JAMA 2003, 289:454-465.

31. Lexchin J, Bero LA, Djulbegovic B, Clark O: Pharmaceutical industry sponsorship and research outcome and quality: systematic review. BMJ 2003, 326:1167-1170.

32. Sismondo S: Pharmaceutical company funding and its consequences: a qualitative systematic review. Contemp Clin Trials 2008, 29:109-113.

33. Hartmann M: Impact assessment of the European Clinical Trials Directive: a longitudinal, prospective, observational study analyzing patterns and trends in clinical drug trial applications submitted since 2001 to regulatory agencies in six EU countries. Trials 2012, 13:53.

34. Berendt L, Hakansson C, Bach KF, Dalhoff K, Andreasen PB, Petersen LG, Andersen E, Poulsen HE: Effect of European Clinical Trials Directive on academic drug trials in Denmark: retrospective study of applications to the Danish Medicines Agency 1993-2006. BMJ 2008, 336:33-35.

35. Jowett SM, Macleod J, Wilson S, Hobbs FD: Research in primary care: extent of involvement and perceived determinants among practitioners from one English region. Br J Gen Pract 2000, 50:387-389. 
36. Raftery J, Bryant J, Powell J, Kerr C, Hawker S: Payment to healthcare professionals for patient recruitment to trials: systematic review and qualitative study. Health Technol Assess 2008, 12:10.

37. Andersen M, Kragstrup J, Sondergaard J: How conducting a clinical trial affects physicians' guideline adherence and drug preferences. JAMA 2006, 295:2759-2764.

38. Nordic Medico-Statistical Committee/Nordic Social Statistical Committee: Medicines Consumption in the Nordic Countries 2004-2008.

[http://www.nom-nos.dk].

39. Krumholz SD, Egilman DS, Ross JS: Study of neurontin: titrate to effect, profile of safety (STEPS) trial: a narrative account of a gabapentin seeding trial. Arch Intern Med 2011, 171:1100-1107.

40. Hill KP, Ross JS, Egilman DS, Krumholz HM: The ADVANTAGE seeding trial: a review of internal documents. Ann Intern Med 2008, 149:251-258.

41. Altman DG: Practical Statistics for Medical Research. London, UK: Chapman \& Hall; 1991

42. Holtedahl KA, Meland E, Klovning A: [New critical questions to drug industry]. Tidsskr Nor Laegeforen 2002, 122:2309.

43. Meineche-Schmidt V, Juhl HH, Ostergaard JE, Luckow A, Hvenegaard A: Costs and efficacy of three different esomeprazole treatment strategies for long-term management of gastro-oesophageal reflux symptoms in primary care. Aliment Pharmacol Ther 2004, 19:907-915.

44. Beglinger C, Thalmann C, Szucs T, Michetti P: [Long-term treatment of patients with symptomatic gastroesophageal reflux disease comparing costs and efficacy over 6 months of treatment with Nexium On-Demand Treatment or Nexium continuous treatment. An open, randomised multi-center study]. Praxis (Bern 1994) 2006, 95:483-487.

doi:10.1186/1745-6215-14-162

Cite this article as: Brænd et al:: Clinical drug trials in general practice: a 10-year overview of protocols. Trials 2013 14:162.

\section{Submit your next manuscript to BioMed Central and take full advantage of:}

- Convenient online submission

- Thorough peer review

- No space constraints or color figure charges

- Immediate publication on acceptance

- Inclusion in PubMed, CAS, Scopus and Google Scholar

- Research which is freely available for redistribution

Submit your manuscript at www.biomedcentral.com/submit
C Biomed Central 